(1)

CrossMark

\title{
Interstitial lung disease in primary immunodeficiency: towards a brighter future
}

\author{
John R. Hurst ${ }^{1}$ and Klaus Warnatz ${ }^{2,3}$, on behalf of the ERS eGLILDnet Clinical \\ Research Collaboration ${ }^{4}$
}

Affiliations: ${ }^{1} \mathrm{UCL}$ Respiratory, University College London, London, UK. ${ }^{2}$ Dept of Rheumatology and Clinical Immunology, Medical Center - University of Freiburg, Faculty of Medicine, University of Freiburg, Freiburg, Germany. ${ }^{3}$ Center for Chronic Immunodeficiency, Medical Center - University of Freiburg, Faculty of Medicine, University of Freiburg, Freiburg, Germany. ${ }^{4} \mathrm{~A}$ list of the ERS eGLILDnet Clinical Research Collaborators can be found in the acknowledgements section.

Correspondence: John R. Hurst, University College London, Centre for Inflammation and Tissue Repair, UCL Respiratory Medicine, London, NW3 2PF, UK. E-mail: j.hurstQucl.ac.uk

@ERSpublications

The ERS eGLILDnet CRC is working for better care and research for people affected by GLILD http://bit.ly/37WZGdd

Cite this article as: Hurst JR, Warnatz K. Interstitial lung disease in primary immunodeficiency: towards a brighter future. Eur Respir J 2020; 55: 2000089 [https://doi.org/10.1183/13993003.00089-2020].

Lung disease is a frequent clinical manifestation in people living with primary immunodeficiency diseases, the most prevalent of which are common variable immunodeficiency disorders (CVID). CVID is primarily characterised by antibody deficiency, but recent definitions and diagnostic criteria recognise a much more complex pattern of immunological defects [1]. CVID can be classified into two major clinical phenotypes. One group experiences infection as the only major clinical manifestation, whilst the other present a variety of lymphoproliferative, inflammatory and/or autoimmune complications. The most frequent consequences in the lung of CVID are acute infections, and secondary airway complications of infection, such as bronchiectasis. However, up to $15 \%$ of patients with CVID develop an interstitial lung disease $[2,3]$. Infections and bronchiectasis are primarily driven by antibody deficiency, but CVID associated interstitial lung disease (CVID-ILD) is best considered part of a systemic immune dysregulatory process [4] such that people with CVID-ILD often have splenomegaly, lymphadenopathy and autoimmune cytopenias [5-7]. With an EU population of 747 million, we estimate there are up to 30000 people living with CVID in Europe, and thus 4500 with CVID-ILD. Whilst people with "infection only" CVID can now expect a near normal life expectancy [8], those with systemic immune dysregulation including CVID-ILD often have a much more complicated course. CVID-ILD increases morbidity and mortality in CVID [9], although the outcome is now recognised to be more variable than originally reported [10].

The terminology in use for ILD in CVID is complex and there is no consensus. The term granulomatous-lymphocytic interstitial lung disease (GLILD) is in common use but there need not be granuloma. We previously proposed the following working definition of GLILD: "GLILD is a distinct clinico-radio-pathological ILD occurring in patients with CVID, associated with a lymphocytic infiltrate and/or granuloma in the lung, and in whom other conditions have been considered and where possible excluded" [11]. Lung pathology and its radiological correlate in CVID-ILD/GLILD are heterogeneous including frequent ground-glass opacities due to lymphocytic interstitial pneumonitis, organising 
FIGURE 1 Typical computed tomography (CT) chest scan from a patient with common variable immunodeficiency disorder at the time of initial diagnosis with granulomatouslymphocytic interstitial lung disease. The patient was asymptomatic but had impairment in gas transfer. The CT also demonstrated splenomegaly.

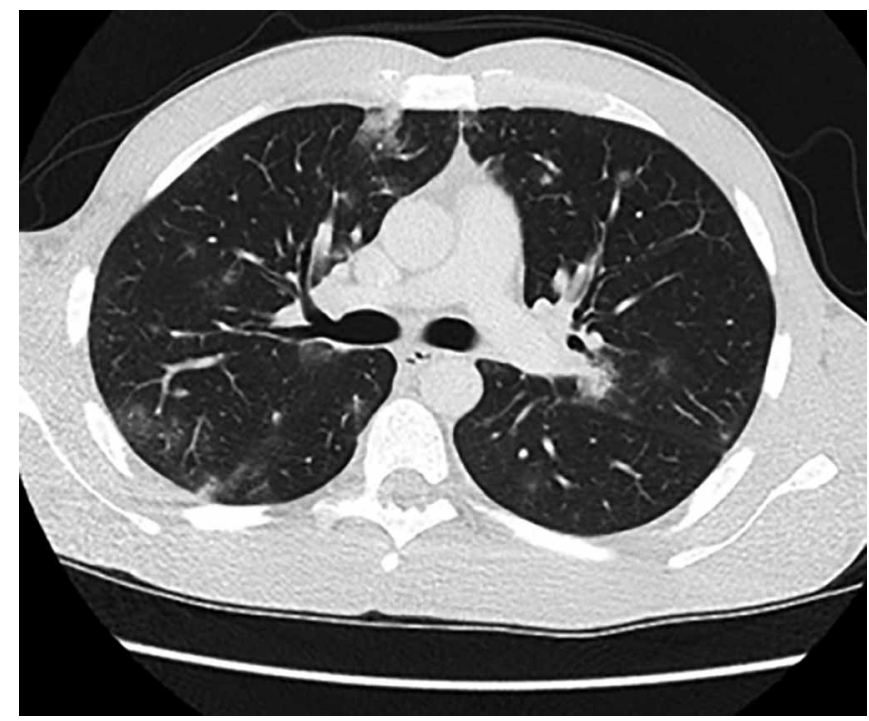

pneumonia, and larger, often bronchocentric nodules, often in the context of background bronchiectasis (figure 1) $[12,13]$. No pathogen has been identified and the mechanism is likely immune dysregulation. Histology is characterised by combined T- and B-lymphocytic infiltrates, partly creating tertiary lymphoid structures within the lung and associated with increased levels of the B-cell activating factor BAFF [14]. The frequent coincidence of lymphadenopathy raises the important differential diagnoses of sarcoid and lymphoma. Associated laboratory abnormalities include elevated soluble interleukin-2 receptor, $\beta 2$ microglobulin and neopterin, together with the hypogammaglobulinaemia that is the hallmark of CVID and which facilitates differentiation from sarcoidosis in previously undiagnosed patient with CVID-ILD.

Managing patients with rare diseases is challenging in the absence of robust evidence. Many questions about the pathogenesis, diagnosis and management of GLILD remain. Is a lung biopsy necessary? What constitutes optimal treatment given that the available evidence, such as it is, remains based on case reports and small case series [15-18]? The relevance of B-cells in the pathogenesis of CVID-ILD/GLILD is underlined by the reported effectiveness of rituximab [19]. New treatments in ILD such as nintedanib and pirfenidone have not been tested in CVID-ILD/GLILD, although fibrosis is not a prominent feature of CVID-ILD/GLILD.

To understand current management, we conducted a survey in the UK to assess diagnostic work up, and how clinicians were making treatment decisions including starting criteria, choice of drug, and endpoints such as symptoms, lung function and radiology [11]. The large (observational) European STILPAD (Study of interstitial lung disease in primary antibody deficiency) study will shortly report retrospective results, adding to the existing literature. Such evidence is urgently required to better treat people with this condition.

With considerable progress in understanding and treating other ILD, it becomes increasingly frustrating and unacceptable not to address the challenges in managing CVID-ILD/GLILD. To this end, we are pleased to announce the launch of a European Respiratory Society Clinical Research Collaboration (ERS CRC) to address CVID-ILD/GLILD, called eGLILDnet. As has been previously described, ERS CRCs are established to promote the exchange of research ideas among clinicians and scientists in Europe and more widely and to plan, conduct, evaluate and publish clinical and translational studies [20].

eGLILDnet will create a multi-professional network that is designed to establish a virtual multidisciplinary team for case-based expert discussion in collaboration with ERN RITA, and a registry for cases (ERN RITA is the European Reference Network for immunodeficiency, autoinflammatory and autoimmune disease). We will go on to complete a research prioritisation exercise, ultimately advocating for and delivering necessary practice changing research in CVID-ILD/GLILD. Only by wide, transparent, multi-professional and trans-disciplinary collaboration across Europe can we strive to deliver a brighter future for those affected by CVID-ILD/GLILD. Like all CRCs, eGLILDnet is a collaboration of patients, clinicians, scientists and industry, and designed to support training through the involvement of early career researchers.

How can you help? If you would like to join us, please contact the co-chairs. You can follow updates on Twitter (@glildnet) or at our website (www.ersnet.org/research/e-glildnet-a-european-granulomatouslymphocytic-interstitial-lung-disease-network). 
And practically? Always remember to measure serum immunoglobulins in people with SPUR (severe, persistent, unusual or recurrent) infections, bronchiectasis and interstitial lung disease, particularly sarcoidosis, to avoid missing primary immunodeficiency.

Support statement: This work was funded by the European Respiratory Society (grant: ERS CRC eGLILDnet).

Conflict of interest: J.R. Hurst reports grants, personal fees and non-financial support from companies that make immunoglobulin products, and medicines to treat respiratory diseases, outside the submitted work. K. Warnatz reports grants from $\mathrm{BMBF}$ and European Respiratory Society, grants and non-financial support (study medication) from Bristol Myers Squibb (BMS), during the conduct of the study; grants and personal fees from Biotest and CSL Behring, personal fees from Baxter/Shire/Takeda, Roche and Octapharma, outside the submitted work.

The ERS eGLILDnet Clinical Research Collaboration: Tiago M. Alfaro, Pneumology Unit, Centro Hospital e Universitário de Coimbra, Coimbra, Portugal and Centre of Pneumology, Faculty of Medicine, University of Coimbra, Coimbra, Portugal; Ulrich Baumann, Paediatric Pulmonology, Allergy and Neonatology, Hannover Medical School, Hanover, Germany; Anne Bergeron, Université de Paris, APHP Hôpital Saint Louis, UMR 1153 CRESS, Paris, France; Siobhan O. Burns, Institute of Immunity and Transplantation, University College London, London, UK, Dept of Immunology, Royal Free London NHS Foundation Trust, London, UK; Alison Condliffe, Dept of Infection, Immunity and Cardiovascular Diseases, University of Sheffield Medical School, Sheffield, UK; Andrew R. Gennery, Translational and Clinical Research Institute, Newcastle University and Great North Children's Hospital, Newcastle upon Tyne, UK; Filomeen Haerynck, Dept of Pediatric Pulmonology and Immunology, Centre for Primary Immune deficiency Ghent, PID research lab, Ghent University Hospital, Belgium; Joseph Jacob, UCL Respiratory, University College London and Centre for Medical Image Computing, University College London, London, UK; Stephen Jolles, Immunodeficiency Centre for Wales, University Hospital of Wales, Cardiff, UK; Marion Malphettes, Dept of Clinical Immunology, Hôpital Saint-Louis, Assistance Publique Hôpitaux de Paris (APHP), Paris, France, EA3518, Université Paris Diderot, Paris, France; Véronique Meignin, Dept of Pathology, Hôpital Saint-Louis, Assistance Publique Hôpitaux de Paris (APHP), Paris, France, Tomas Milota, Dept of Immunology, Second Faculty of Medicine Charles University and Motol University Hospital, Prague, Czech Republic; Antje Prasse, Dept of Pulmonology, Hannover Medical School and DZL BREATH, Hannover, Germany and Fraunhofer ITEM, Hannover, Germany; Isabella Quinti, Dept of Molecular Medicine, Sapienza University of Rome, Rome, Italy; Elisabetta Renzoni, Royal Brompton Hospital, London, UK; Daiana Stolz, Clinic for Respiratory Medicine and Pulmonary Cell Research, University Hospital Basel, Basel, Switzerland; Annick A.J.M. van de Ven, Depts of Internal Medicine and Allergology, Rheumatology and Clinical Immunology, University Medical Center Groningen, the Netherlands.

\section{References}

1 Bonilla FA, Barlan I, Chapel H, et al. International Consensus Document (ICON): common variable immunodeficiency disorders. J Allergy Clin Immunol Pract 2016; 4: 38-59.

2 Verma N, Grimbacher B, Hurst JR. Lung disease in primary antibody deficiency. Lancet Respir Med 2015; 3: 651-660.

3 van de Ven AA, de Jong PA, Hoytema van Konijnenburg DP, et al. Airway and interstitial lung disease are distinct entities in paediatric common variable immunodeficiency. Clin Exp Immunol 2011; 165: 235-242.

4 Schussler E, Beasley MB, Maglione PJ. Lung disease in primary antibody deficiencies. J Allergy Clin Immunol Pract 2016; 4: 1039-1052.

5 Hartono S, Motosue MS, Khan S, et al. Predictors of granulomatous lymphocytic interstitial lung disease in common variable immunodeficiency. Ann Allergy Asthma Immunol 2017; 118: 614-620.

6 Feuille EJ, Anooshiravani N, Sullivan KE, et al. Autoimmune cytopenias and associated conditions in CVID: a report from the USIDNET Registry. J Clin Immunol 2018; 38: 28-34.

7 Mannina A, Chung $\mathrm{JH}$, Swigris JJ, et al. Clinical predictors of a diagnosis of common variable immunodeficiency-related granulomatous-lymphocytic interstitial lung disease. Ann Am Thorac Soc 2016; 13: 1042-1049.

8 Resnick ES, Moshier EL, Godbold JH, et al. Morbidity and mortality in common variable immune deficiency over 4 decades. Blood 2012; 119: 1650-1657.

9 Bates CA, Ellison MC, Lynch DA, et al. Granulomatous-lymphocytic lung disease shortens survival in common variable immunodeficiency. J Allergy Clin Immunol 2004; 114: 415-421.

10 Maglione PJ, Overbey JR, Cunningham-Rundles C. Progression of common variable immunodeficiency interstitial lung disease accompanies distinct pulmonary and laboratory findings. J Allergy Clin Immunol Pract 2015; 3: 941-950.

11 Hurst JR, Verma N, Lowe D, et al. British Lung Foundation/United Kingdom Primary Immunodeficiency Network Consensus Statement on the definition, diagnosis, and management of granulomatous-lymphocytic interstitial lung disease in common variable immunodeficiency disorders. J Allergy Clin Immunol Pract 2017; 5: 938-945.

12 Prasse A, Kayser G, Warnatz K. Common variable immunodeficiency-associated granulomatous and interstitial lung disease. Curr Opin Pulm Med 2013; 19: 503-509.

13 Patel S, Anzilotti C, Lucas M, et al. Interstitial lung disease in patients with common variable immunodeficiency disorders: several different pathologies? Clin Exp Immunol 2019; 198: 212-223.

14 Maglione PJ, Gyimesi G, Cols M, et al. BAFF-driven B cell hyperplasia underlies lung disease in common variable immunodeficiency. JCI Insight 2019; 4: e122728.

15 Chase NM, Verbsky JW, Hintermeyer MK, et al. Use of combination chemotherapy for treatment of granulomatous and lymphocytic interstitial lung disease (GLILD) in patients with common variable immunodeficiency (CVID). J Clin Immunol 2013; 33: 30-39.

16 Cinetto F, Scarpa R, Pulvirenti F, et al. Appropriate lung management in patients with primary antibody deficiencies. Expert Rev Respir Med 2019; 13: 823-838. 
17 Deyà-Martínez $\mathrm{A}$, Esteve-Solé $\mathrm{A}$, Vélez-Tirado $\mathrm{N}$, et al. Sirolimus as an alternative treatment in patients with granulomatous-lymphocytic lung disease and humoral immunodeficiency with impaired regulatory T cells. Pediatr Allergy Immunol 2018; 29: 425-432.

18 Bucciol G, Petrone A, Putti MC. Efficacy of mycophenolate on lung disease and autoimmunity in children with immunodeficiency. Pediatr Pulmonol 2017; 52: E73-E76.

19 Pecoraro A, Crescenzi L, Galdiero MR, et al. Immunosuppressive therapy with rituximab in common variable immunodeficiency. Clin Mol Allergy 2019; 17: 9.

20 Brightling C, Genton C, Bill W, et al. ERS Clinical Research Collaborations: underpinning research excellence. Eur Respir J 2018; 52: 1801534. 